\title{
Difference between the Time Intervals in the Special Relativistic Theory Calculated with the Aid of an Invariance Property between the Mechanical Expressions
}

\author{
Stanisław Olszewski \\ Institute of Physical Chemistry, Polish Academy of Sciences, Warsaw, Poland \\ Email: olsz@ichf.edu.pl
}

How to cite this paper: Olszewski, S (2021) Difference between the Time Intervals in the Special Relativistic Theory Calculated with the Aid of an Invariance Property between the Mechanical Expressions. Journal of Modern Physics, 12, 2031-2040. https://doi.org/10.4236/jmp.2021.1214116

Received: November 24, 2021

Accepted: December 28, 2021

Published: December 31, 2021

Copyright (อ 2021 by author(s) and Scientific Research Publishing Inc. This work is licensed under the Creative Commons Attribution International License (CC BY 4.0).

http://creativecommons.org/licenses/by/4.0/

\begin{abstract}
In the relativistic mechanics, we obtain a difference between the time scale of a one-dimensional motion having a larger velocity and the time scale of a similar motion with a lower velocity. This calculation does involve usually also the differences of parameters other than time. Basing on the invariance of a pair of the mechanical parameters, it can be shown that the difference of two scales of time can be attained independently from the differences of the other physical parameters.
\end{abstract}

\section{Keywords}

Time in the Special Relativity Theory, Relativistic Invariance of Some Special Differences of the Mechanical Parameter

\section{Introduction}

In principle, any calculation of a change of the scale of some mechanical parameter in the special relativistic theory is accompanied by an insight into the scale changes belonging also to other parameters than that taken into account [1]. But recently an invariance of the differences of parameters belonging to different mechanical scales has been pointed out [2]. Our aim became to demonstrate that this situation allows us to estimate the size change of a single mechanical parameter alone. The scale of time has been chosen as our basic example.

In the first step, we present the Lorentz transformation of the variables pair $t, x$ into the pair $t^{\prime}, x^{\prime}$ [2]. Next the intervals $t^{\prime}-t$ and $x^{\prime}-x$ are calculated in terms of $t$ and $x$. Finally the quanta of the electric capacitance in the hydrogen 
atom and their change with the quantum number are examined.

\section{Lorentz Transformation and the Invariant Expressions for the Mechanical Parameters}

If the Lorentz transformation is specified for the variables pair $(t, x)$ going into the pair $\left(t^{\prime}, x^{\prime}\right)$, the corresponding transformation formulae are:

$$
t^{\prime}=t \frac{1}{\sqrt{1-\frac{v^{2}}{c^{2}}}}-\frac{x \frac{v}{c}}{\sqrt{1-\frac{v^{2}}{c^{2}}}}
$$

and

$$
x^{\prime}=-\frac{t \frac{v}{c}}{\sqrt{1-\frac{v^{2}}{c^{2}}}}+\frac{x}{\sqrt{1-\frac{v^{2}}{c^{2}}}} .
$$

The $t$ and $t^{\prime}$ are the time coordinates, the $x$ and $x^{\prime}$ are coordinates of position in the particle one-dimensional motion along the axis $x$, so the changes of two parameters are involved. The $v$ is a speed between the system labelled by $\left(t^{\prime}, x^{\prime}\right)$ and that labelled by $(t, x)$. We obtain for the difference [2]

$$
\begin{aligned}
t^{\prime 2}-x^{\prime 2} & =\left[t^{2}-2 x t \frac{v}{c}+x^{2} \frac{v^{2}}{c^{2}}\right] \frac{1}{1-\frac{v^{2}}{c^{2}}}-\left[t^{2} \frac{v^{2}}{c^{2}}-2 x t \frac{v}{c}+x^{2}\right] \frac{1}{1-\frac{v^{2}}{c^{2}}} \\
& =\left[t^{2}\left(1-\frac{v^{2}}{c^{2}}\right)-x^{2}\left(1-\frac{v^{2}}{c^{2}}\right)\right] \frac{1}{1-\frac{v^{2}}{c^{2}}}=t^{2}-x^{2} .
\end{aligned}
$$

The result in (3) indicates the invariant behaviour of the difference

$$
t^{\prime 2}-x^{\prime 2}=t^{2}-x^{2}
$$

upon the Lorentz transformation formulae in (1) and (2). From (3a) it comes out that

$$
t^{\prime 2}-t^{2}=x^{\prime 2}-x^{2}=\Delta t\left(t^{\prime}+t\right)=\Delta x\left(x^{\prime}+x\right)
$$

on condition we put

$$
\Delta t=t^{\prime}-t
$$

and

$$
\Delta x=x^{\prime}-x .
$$

Our aim is to point out that the above properties concerning the transformed and non-transformed parameters of $t$ and $x$ can lead to a simple reference between $t^{\prime}$ and $t$.

\section{The Difference of $t^{\prime}$ and $t$ Represented as a Function of Some Remainder Parameters}

We multiply Equation (1) by $t$ and Equation (2) by $x$. A simple rearrangement of 
terms leads to the formulae:

$$
t^{\prime} t-\frac{t^{2}}{\left(1-\frac{v^{2}}{c^{2}}\right)^{1 / 2}}=-\frac{v x t}{\sqrt{1-\frac{v^{2}}{c^{2}}}} \frac{1}{c}
$$

and

$$
x^{\prime} x-\frac{x^{2}}{\left(1-\frac{v^{2}}{c^{2}}\right)^{1 / 2}}=-\frac{v t x}{\sqrt{1-\frac{v^{2}}{c^{2}}}} \frac{1}{c} .
$$

The difference of (5) and (6) leads to the result:

$$
t^{\prime} t-x^{\prime} x-\frac{t^{2}-x^{2}}{\left(1-\frac{v^{2}}{c^{2}}\right)^{1 / 2}}=0
$$

or with the aid of (3a) to the formula:

$$
t^{\prime} t-x^{\prime} x=\frac{t^{2}-x^{2}}{\left(1-\frac{v^{2}}{c^{2}}\right)^{1 / 2}}=\frac{t^{\prime 2}-x^{\prime 2}}{\left(1-\frac{v^{2}}{c^{2}}\right)^{1 / 2}}
$$

The result in (8) is dictated by the property (3a). The calculations done farther in the paper refer to the non-primed coordinates system

Another approach to the rearrangement of terms in (8) gives:

$$
t^{\prime 2}-t^{2}=x^{\prime 2}-x^{2} \text {. }
$$

By taking into account (4a) and (4b) we have:

$$
\begin{aligned}
& t^{\prime 2}-t^{2}=\left(t^{\prime}-t\right)\left(t^{\prime}+t\right)=\Delta t(2 t+\Delta t), \\
& x^{\prime 2}-x^{2}=\left(x^{\prime}-x\right)\left(x^{\prime}+x\right)=\Delta x(2 x+\Delta x),
\end{aligned}
$$

but in virtue of (9):

$$
\Delta t(2 t+\Delta t)=\Delta x(2 x+\Delta x) .
$$

The left-hand side of (8) can be transformed into

$$
(t+\Delta t) t-(x+\Delta x) x=t^{2}-x^{2}+t \Delta t-x \Delta x,
$$

so

$$
t \Delta t-x \Delta x=\frac{t^{2}-x^{2}}{\left(1-\frac{v^{2}}{c^{2}}\right)^{1 / 2}}-\left(t^{2}-x^{2}\right)=\left(t^{2}-x^{2}\right)\left[\frac{1}{\left(1-\frac{v^{2}}{c^{2}}\right)^{1 / 2}}-1\right]
$$

indicating a positive value of (11a) on condition $t>x$.

An effect of (4a) and (4b) leads together with the relation (3) to the formula:

$$
\begin{aligned}
t^{2}-x^{\prime 2} & =(t+\Delta t)^{2}-(x+\Delta x)^{2} \\
& =t^{2}-x^{2}+2(t \Delta t-x \Delta x)+(\Delta t)^{2}-(\Delta x)^{2} \\
& =t^{2}-x^{2}
\end{aligned}
$$


so the last step of (12) gives

$$
2(t \Delta t-x \Delta x)=-\left[(\Delta t)^{2}-(\Delta x)^{2}\right]
$$

or

$$
(\Delta x)^{2}+2 x \Delta x-(\Delta t)^{2}-2 t \Delta t=0 .
$$

\section{An Equation for the Interval $\Delta t$ and Its Properties}

Because of (11a) we have

$$
t \Delta t-\left(t^{2}-x^{2}\right)\left[\frac{1}{\left(1-\left(\frac{v}{c}\right)^{2}\right)^{1 / 2}}-1\right]=x \Delta x,
$$

therefore

$$
\frac{t}{x} \Delta t-\frac{1}{x}\left(t^{2}-x^{2}\right)\left[\frac{1}{\left[1-\left(\frac{v}{c}\right)^{2}\right]^{1 / 2}}-1\right]=\Delta x .
$$

A final equation for the interval $\Delta t$ is obtained from the relations (11a), (13) and (14a):

$$
\begin{aligned}
& 2(t \Delta t-x \Delta x)=2\left(t^{2}-x^{2}\right)\left[\frac{1}{\left[1-\left(\frac{v}{c}\right)^{2}\right]^{1 / 2}}-1\right]=-(\Delta t)^{2}+(\Delta x)^{2} \\
& =-(\Delta t)^{2}+\left\{\frac{t}{x} \Delta t-\frac{1}{x}\left(t^{2}-x^{2}\right)\left[\frac{1}{\left[1-\left(\frac{v}{c}\right)^{2}\right]^{1 / 2}}-1\right]\right\}^{2} \\
& =-(\Delta t)^{2}+\left(\frac{t}{x}\right)^{2}(\Delta t)^{2}-\frac{2}{x^{2}} t \Delta t\left(t^{2}-x^{2}\right)\left[\frac{1}{\left[1-\left(\frac{v}{c}\right)^{2}\right]^{1 / 2}}-1\right] \\
& +\frac{1}{x^{2}}\left(t^{2}-x^{2}\right)^{2}\left[\frac{1}{\left[1-\left(\frac{v}{c}\right)^{2}\right]^{1 / 2}}-1\right]^{2}
\end{aligned}
$$


In effect the equation coupling the pairs $\left(t^{\prime}, x^{\prime}\right)$ and $(t, x)$ becomes

$$
\begin{aligned}
& {\left[\left(\frac{t}{x}\right)^{2}-1\right](\Delta t)^{2}-\frac{2 t}{x^{2}}\left(t^{2}-x^{2}\right)\left[\frac{1}{\left[1-\left(\frac{v}{c}\right)^{2}\right]^{1 / 2}}-1\right] \Delta t} \\
& +\frac{\left(t^{2}-x^{2}\right)^{2}}{x^{2}}\left[\frac{1}{\left[1-\left(\frac{v}{c}\right)^{2}\right]^{1 / 2}}-1\right]-2\left(t^{2}-x^{2}\right)\left\{\frac{1}{\left[1-\left(\frac{v}{c}\right)^{2}\right]^{1 / 2}}-1\right\}=0 .
\end{aligned}
$$

We divide (16) by $\left[\left(\frac{t}{x}\right)^{2}-1\right]$ and get the equation:

$$
\begin{aligned}
& (\Delta t)^{2}-2 t \Delta t\left[\frac{1}{\left[1-\left(\frac{v}{c}\right)^{2}\right]^{1 / 2}}-1\right]+\left(t^{2}-x^{2}\right)\left[\frac{1}{\left[1-\left(\frac{v}{c}\right)^{2}\right]^{1 / 2}}-1\right]^{2} \\
& -2 x^{2}\left[\frac{1}{\left[1-\left(\frac{v}{c}\right)^{2}\right]^{1 / 2}}-1\right]=0
\end{aligned}
$$

which gives

$$
(\Delta t)^{2}-2 t \Delta t[]+t^{2}[]^{2}=x^{2}[]^{2}+2 x^{2}[]
$$

or

$$
\{\Delta t-t[]\}^{2}=x^{2}[]^{2}+2 x^{2}[] .
$$

The contents of the square brackets in (17a) and (18) is represented in (17).

Taking the square root of (18) we obtain:

$$
\Delta t-t\left[\frac{1}{\left[1-\left(\frac{v}{c}\right)^{2}\right]^{1 / 2}}-1\right]=x\left\{[]^{2}+2[]\right\}^{1 / 2} .
$$

We divide both sides of (19) by $t$.

$$
\frac{\Delta t}{t}-\left[\frac{1}{\left[1-\left(\frac{v}{c}\right)^{2}\right]^{1 / 2}}-1\right]=\frac{x}{t}\left\{[]^{2}+2[]\right\}^{1 / 2} .
$$

From this equation we have: 


$$
\frac{\Delta t}{t}=\frac{1}{\left[1-\left(\frac{v}{c}\right)^{2}\right]^{1 / 2}}-1+\frac{x}{t}\left\{[]^{2}+2[]\right\}^{1 / 2} .
$$

We see from (21) that the ratio $\Delta t / t$ is a sum of a constant dependent solely on $v / c$ increased by the ratio $x / t$ multiplied by another constant term depend solely on $v / c$.

The next step concerns solution of the equation for $\Delta x$. We obtain from (13a)

$$
(\Delta x)^{2}+2 x \Delta x-\left[(\Delta t)^{2}+2 t \Delta t\right]=(\Delta x)^{2}+2 x \Delta x-\Delta t[2 t+\Delta t]=0 .
$$

This gives

$$
\Delta x_{1,2}=\frac{-b \pm \sqrt{\Delta}}{2 a}
$$

where

$$
\begin{gathered}
b=2 x, \\
\Delta=b^{2}-4 a d, \\
a=1, \\
-d=\Delta t[2 t+\Delta t] .
\end{gathered}
$$

The solution of (22) gives:

$$
\begin{aligned}
\Delta x_{1,2} & =\frac{-2 x \pm \sqrt{4 x^{2}+8 t \Delta t+4(\Delta t)^{2}}}{2}=-x \pm \sqrt{x^{2}+2 t \Delta t+(\Delta t)^{2}} \\
& =-x \pm x \sqrt{1+2 \frac{t \Delta t}{x^{2}}+\frac{(\Delta t)^{2}}{x^{2}}} .
\end{aligned}
$$

Assuming $\Delta x$ to be a small number the square root in (28) is taken next only with a positive sign. We obtain $(\Delta x+x)^{2}=x^{2}+2 t \Delta t+(\Delta t)^{2}$ or

$$
(\Delta x)^{2}+2 x \Delta x=2 t \Delta t+(\Delta t)^{2} .
$$

On the basis of the result in (21) we obtain for the right-hand side of (29) the expression dependent solely on $t$ and $x$.

$$
2 t \Delta t+(\Delta t)^{2}=2 t^{2}\left[\frac{1}{\left[1-\left(\frac{v}{c}\right)^{2}\right]^{1 / 2}}-1\right]+2 t x\left\{[]^{2}+2[]\right\}^{1 / 2}+(\Delta t)^{2} .
$$

The term $(\Delta t)^{2}$ can be readily obtained from (21) as a function of $t$ and $x$.

\section{The Calculation of $\Delta x=x^{\prime}-x$}

We do it by taking into account the relation (9):

$$
\begin{aligned}
t^{\prime 2}-t^{2} & =\left(t^{\prime}-t\right)\left(t^{\prime}+t\right)=\Delta t(2 t+\Delta t) \\
& =x^{\prime 2}-x^{2}=\left(x^{\prime}-x\right)\left(x^{\prime}+x\right)=\Delta x(2 x+\Delta x) .
\end{aligned}
$$


We obtain:

$$
\frac{\Delta x}{x}+1=\sqrt{\frac{(\Delta x+x)^{2}}{x^{2}}}=\sqrt{\frac{x^{2}+2 x \Delta x+(\Delta x)^{2}}{x^{2}}}=\sqrt{1+\frac{\Delta t(2 t+\Delta t)}{x^{2}}}
$$

where expression $\Delta t(2 t+\Delta t)$ is represented by (30).

It seems interesting to compare both terms entering the product entering the last square root in (32). We have [see (19)]

$$
\Delta t=t\left[\frac{1}{\left(1-\frac{v^{2}}{c^{2}}\right)^{1 / 2}}-1\right]+x\left\{[]^{2}+2[]\right\}^{1 / 2}
$$

whereas

$$
2 t+\Delta t=t\left[\frac{1}{\left(1-\frac{v^{2}}{c^{2}}\right)^{1 / 2}}+1\right]+x\left\{[]^{2}+2[]\right\}^{1 / 2} .
$$

In effect:

$$
\Delta t(2 t+\Delta t)=\frac{t^{2}}{1-\frac{v^{2}}{c^{2}}}-t^{2}+2 t \frac{x}{\left(1-\frac{v^{2}}{c^{2}}\right)^{1 / 2}}\left\{[]^{2}+2[]\right\}^{1 / 2}+x^{2}\left\{[]^{2}+2[]\right\} .
$$

Therefore

$$
\frac{\Delta x}{x}+1=\left\{1+\frac{t^{2}}{x^{2}}\left[\frac{1}{1-\frac{v^{2}}{c^{2}}}-1\right]+\frac{2 t}{x} \frac{1}{\left(1-\frac{v^{2}}{c^{2}}\right)^{1 / 2}}\left\{[]^{2}+2[]\right\}^{1 / 2}+[]^{2}+2[]\right\}
$$

The expressions entering the empty brackets in (17a)-(21) as well as in (30) and (33)-(36) are fully represented in Equation (17).

\section{Example of Distance $x$ Representing the Quanta of the Electric Capacitance and Their Change}

An example of $x$ and $\Delta x$ can be presented by considering the electric capacitance $C$ of the condenser. A reference to $C$ and the charge $q$ of the condenser is given by the formula [3]:

$$
V=\frac{q}{C}
$$

where $V$ is the potential difference. On the other side, when the potential difference (37) is applied to the resistance $R$, the current intensity $i$ produced by $V$ and $R$ becomes

$$
i=\frac{V}{R} .
$$


Let us assume that $i$ is representing the electric current done along the $n$-th electron orbit about the nucleus of the hydrogen atom. Then

$$
R i_{n}=R \frac{e}{T_{n}}=\frac{q}{C} .
$$

Here [4]

$$
T_{n}=\frac{2 \pi \hbar^{3} n^{3}}{e^{4} m}
$$

is the time period of the electron motion along the $n$th orbit, $m$ is the electron mass;

$$
q=e
$$

is the absolute value of the electron charge. Then it can be shown that $R$ is the quantum parameter [5]

$$
R=\frac{h}{e^{2}}
$$

valid for all orbits $n$ in the atom. It is worth to note that the $R$ size in (42) is equal to the resistance characteristic for the integer quantum Hall effect; see e.g. [6].

By taking into account (39), (41) and (42) we obtain for $C$ :

$$
C_{n}=\frac{T_{n}}{R}=\frac{2 \pi \hbar^{3} n^{3}}{e^{4} m} \frac{e^{2}}{h}=\frac{\hbar^{2} n^{3}}{e^{2} m} .
$$

Evidently the lowest quantum of the capacitance $C_{n}$ corresponds to the orbit $n=1$ :

$$
C_{1}=\frac{\hbar^{2}}{e^{2} m}=0.53 \times 10^{-8} \mathrm{~cm} .
$$

The size of $C_{1}$ is equal to the radius of the shortest orbit in the hydrogen atom. Further $C_{n}(n>1)$ increase proportionally to $n^{3}$ [see (43)], whereas the orbit length remains proportional to $n^{2}$.

The dimension of $C_{n}$ is

$$
C_{n} \sim \frac{\hbar^{2}}{e^{2} m} \sim \frac{\mathrm{erg}^{2} \cdot \mathrm{sec}^{2}}{\mathrm{erg} \cdot \mathrm{cm} \cdot \mathrm{g}} \sim \mathrm{cm} .
$$

In this way we find that

$$
C_{n} \sim \mathrm{cm} \sim x .
$$

A relativistic change of $x$ modifies the $x$ entering (46) into $x+\Delta x$.

\section{Euler-Lagrange Equation Applied in Examining the Particle Mass}

In [2] an attempt is done to apply the Euler-Lagrange equation in examining the behaviour of the particle mass $m$. A special application may concern the force $F$ acting on moving particle when it is reduced to a single term [3]: 


$$
F=\frac{\mathrm{d}(m \dot{x})}{\mathrm{d} t}=m \frac{\mathrm{d} \dot{x}}{\mathrm{~d} t}+\frac{\mathrm{d} m}{\mathrm{~d} t} \dot{x}=\frac{\mathrm{d} m}{\mathrm{~d} t} \dot{x} .
$$

This holds on condition

$$
\frac{\mathrm{d} \dot{x}}{\mathrm{~d} t}=0, \quad \text { or } \quad \dot{x}=\text { const. }
$$

The Lagrangian function let be [2]:

$$
L=-[m+\varphi(t, x)] \sqrt{1-\dot{x}^{2}}
$$

where $\varphi$ is a time component of the vector potential denoted-for historical reasons-by $-\varphi(t, x)$. The derivative $\frac{\mathrm{d} \varphi}{\mathrm{d} t}$ can be joined with the mass derivative $\frac{\mathrm{d} m}{\mathrm{~d} t}$, so the Euler-Lagrange equation becomes

$$
\frac{\mathrm{d}}{\mathrm{d} t} \frac{\partial L}{\partial \dot{x}}=\left(\frac{\mathrm{d} m}{\mathrm{~d} t}+\frac{\mathrm{d} \varphi}{\mathrm{d} t}\right) \frac{\dot{x}}{\sqrt{1-\dot{x}^{2}}}=\frac{\partial L}{\partial x}=-\frac{\partial \varphi}{\partial x} \sqrt{1-\dot{x}^{2}}
$$

Equation (50) gives:

$$
F=\dot{x} \frac{\mathrm{d} m}{\mathrm{~d} t}=-\frac{\mathrm{d} \varphi}{\mathrm{d} t} \dot{x}-\frac{\partial \varphi}{\partial x}\left(1-\dot{x}^{2}\right)=-\frac{\mathrm{d} \varphi}{\mathrm{d} t} \dot{x}-\frac{\partial \varphi}{\partial x}+\frac{\partial \varphi}{\partial x} \dot{x}^{2} .
$$

Evidently for $\frac{\partial \varphi}{\partial x}>0$ beginning from $\dot{x}=0$ the formula (51) can provide us with $F$ tending to an expression representing a positive $F>0$.

\section{Summary}

In the paper, the problem of a change of the interval $\Delta t$ of a single mechanical variable-namely the time $t$-due to the existence of a non-vanishing velocity $v$ of a moving one-dimensional system has been examined. First a simple dependence on $v$ for the change $\Delta t$ of the variable $t$ is obtained, next a similar dependence is calculated for $\Delta x$ equal to the change of the position coordinate $x$.

\section{Conflicts of Interest}

The author declares no conflicts of interest regarding the publication of this paper.

\section{References}

[1] Sommerfeld, A. (1949) Mechanik. $4^{\text {th }}$ Edition, Akademische Verlagsgesellschaft Geest \& Portig, Leipzig.

[2] Susskind, L. and Friedman, A. (2019) Special Relativity and Classical Field Theory. Prószyński Media, Warszawa. (In Polish)

[3] Jauncey, G.E.M. (1948) Modern Physics. 3rd Edition, Van Nostrand, New York.

[4] Sommerfeld, A. (1931) Atombau und Spektrallinien. Vol. 1, 5th Edition, Vieweg, 
Braunschweig.

[5] Olszewski, S. (2016) Journal of Modern Physics, 7, 1725.

https://doi.org/10.4236/jmp.2016.713155

[6] MacDonald, A.H. (1989) Quantum Hall Effect. A Perspective. Kluwer, Milano. https://doi.org/10.1007/978-94-010-9709-3 